\title{
Potencial de Uso dos Herbicidas Chlorimuron-Ethyl, IMAZEThapyr e Cloransulam-Methyl Na Cultura do FeIJÃo ${ }^{1}$
}

\author{
Potential Use of Chlorimuron-Ethyl, Imazethapyr and Cloransulam-Methyl in Common \\ Bean Crop
}
PROCOPIO, S.O. ${ }^{2}$, BRAZ, A.J.B.P. ${ }^{3}$, BARROSO, A.L.L. ${ }^{3}$, CARGNELUTTI FILHO, A. ${ }^{4}$, CRUVINEL, K.L. ${ }^{5}$, BETTA, M. ${ }^{5}$, BRAZ, G.B.P. ${ }^{5}$, FRAGA FILHO, J.J.S. ${ }^{5}$ e CUNHA JÚNIOR, L.D. ${ }^{5}$

\begin{abstract}
RESUMO - O objetivo deste trabalho foi avaliar a seletividade dos herbicidas chlorimuronethyl, imazethapyr e cloransulam-methyl, aplicados em associação com o herbicida fomesafen, a dez cultivares de feijão. O experimento foi conduzido no municipio de Rio Verde-GO, no plantio das águas, na safra de 2005/2006. O delineamento experimental utilizado foi de blocos ao acaso, em parcelas subdivididas, com três repetições, sendo o fator da parcela principal os tratamentos com herbicidas [fomesafen $\left(225 \mathrm{~g} \mathrm{ha}^{-1}\right)$; fomesafen $\left(225 \mathrm{~g} \mathrm{ha}^{-1}\right)+$ chlorimuron-ethyl (7,5 $\left.\mathrm{g} \mathrm{ha}^{-1}\right)$; fomesafen $\left(225 \mathrm{~g} \mathrm{ha}^{-1}\right)+$ imazethapyr $\left(50 \mathrm{~g} \mathrm{ha}^{-1}\right)$; fomesafen (225 $\left.\mathrm{g} \mathrm{ha}^{-1}\right)$ + cloransulam-methyl $\left(30,24 \mathrm{~g} \mathrm{ha}^{-1}\right)$; e testemunha sem herbicida]; e o da subparcela, os cultivares (BRS Grafite, BRS Horizonte, Pérola, BRS Pitanga, BRS Pontal, BRS Requinte, BRS Supremo, BRS Timbó, BRS Valente e BRS Vereda). As parcelas foram mantidas capinadas, para que não houvesse interferência das plantas daninhas. Os tratamentos com herbicidas foram fitotóxicos; todavia, de forma geral, os sintomas mais severos foram verificados no tratamento que continha chlorimuron-ethyl. A associação de fomesafen com chlorimuron-ethyl provocou as maiores reduções na altura das plantas e no acúmulo de massa da parte aérea das plantas secas, além de prolongar o ciclo de maturação de todos os cultivares. A aplicação isolada de fomesafen reduziu a produtividade de grãos dos cultivares BRS Timbó e BRS Vereda. Quando se adicionou o imazethapyr ao fomesafen, observou-se redução na produtividade dos cultivares BRS Supremo, BRS Timbó e BRS Vereda. A adição de cloransulam-methyl, além de reduzir a produtividade desses três cultivares, também diminuiu a produtividade do cultivar BRS Requinte. Os cultivares Pérola, BRS Pitanga, BRS Pontal e BRS Valente não tiveram suas produtividades reduzidas pela mistura chlorimuron-ethyl + fomesafen. O imazethapyr mostrou potencial para ser utilizado na cultura do feijão.
\end{abstract}

Palavras-chave: Phaseolus vulgaris, fomesafen, inibidores de ALS, sulfonilureia, imidazolinona.

\begin{abstract}
This study aimed to evaluate the selectivity of the herbicides chlorimuron-ethyl, imazethapyr and cloransulam-methyl when applied in association with fomesafen on ten common bean cultivars. The experiment was carried out in Rio Verde-Goias during the growing season of 2005/2006, in a completely randomized block design, with three replicates, in split-plots. The herbicide treatments [fomesafen (225 $\left.\mathrm{g} \mathrm{ha}^{-1}\right)$, fomesafen (225 g ha $\left.{ }^{-1}\right)+$ chlorimuron-ethyl (7.5 $\left.\mathrm{g} \mathrm{ha} \mathrm{a}^{-1}\right)$, fomesafen (225 $\left.\mathrm{g} \mathrm{ha}^{-1}\right)$ + imazethapyr (50 $\left.\mathrm{g} \mathrm{ha}^{-1}\right)$, fomesafen (225 $\left.\mathrm{g} \mathrm{ha} \mathrm{h}^{-1}\right)$ + cloransulam-methyl $\left(30.24 \mathrm{~g} \mathrm{ha}^{-1}\right)$, and control without herbicide] were tested in the plots and the cultivars (BRS Grafite, BRS Horizonte, Pérola, BRS Pitanga, BRS Pontal, BRS Requinte, BRS Supremo, BRS Timbó, BRS Valente and BRS Vereda), in the split-plots. The plots were kept hoed to avoid weed interference. All treatments caused phytotoxicity to the cultivars, but the treatment with chlorimuron-ethyl showed more severe symptoms. This same treatment also resulted in greater reduction of plant height, shoot
\end{abstract}

Recebido para publicação em 6.7.2008 e na forma revisada em 5.6.2009.

2 Pesquisador, Embrapa Tabuleiros Costeiros, 49025-040 Aracaju-SE, <procopio@cpatc.embrapa.br>; ${ }^{3}$ Professor, Faculdade de Agronomia, Universidade de Rio Verde - FESURV, 75901-970 Rio Verde-GO; ${ }^{4}$ Professor do Dep. de Fitotecnia da Universidade Federal de Santa Maria - UFSM, 97105-900 Santa Maria-RS; ${ }^{5}$ Discente, Agronomia - FESURV.

Planta Daninha, Viçosa-MG, v. 27, n. 2, p. 327-336, 2009 
dry matter and an extended maturation cycle. The sole application of fomesafen caused a decrease in grain productivity of BRS Timbó, and BRS Vereda. When fomesafen was applied in a mixture with imazethapyr, a decrease in grain productivity was observed for the cultivars BRS Timbó, BRS Vereda, and BRS Supremo. Cloransulam-methyl addition led to a productivity reduction of these three cultivars and BRS Requinte, as well. The productivity of Pérola, BRS Pitanga, BRS Pontal, and BRS Valente cultivars was not affected by the application of chlorimuron-ethyl + fomesafen. Imazethapyr presented potential to be used in common bean crop.

Keywords: Phaseolus vulgaris, fomesafen, ALS inhibitors, sulfonylurea, imidazolinone.

\section{INTRODUÇÃO}

Além de sua importância econômica, o feijão constitui um dos alimentos básicos da população brasileira e é uma das alternativas de exploração agrícola em pequenas propriedades, de ocupação de mão de obra menos qualificada, e um dos principais produtos fornecedores de proteína na dieta alimentar dos estratos sociais economicamente menos favorecidos (Fuscaldi \& Prado, 2005). Apesar da grande importância dessa cultura, os rendimentos médios obtidos são muito baixos (Silva et al., 2003). Entre os fatores que ocasionam perdas significativas na produtividade do feijão, destaca-se a interferência decorrente da convivência das plantas daninhas com a cultura. A capacidade competitiva dos feijoeiros com as plantas daninhas é pequena, pois eles apresentam metabolismo fotossintético tipo $\mathrm{C} 3$, não respondendo na produção de fotoassimilados em relação às plantas $\mathrm{C} 4$, principalmente em condições de temperaturas mais elevadas e alta intensidade luminosa. O feijoeiro tem porte baixo, ciclo curto, normalmente variando de 70 a 95 dias, sistema radicular superficial e crescimento inicial lento (Cobucci et al., 1996; Burnside et al., 1998). Segundo Victoria Filho \& Godoy Júnior (1978), as plantas daninhas competem significativamente com a cultura do feijão, sobretudo no primeiro terço de desenvolvimento da cultura. De acordo com Lunkes (1997), as perdas na cultura do feijão devido à competição com as plantas daninhas podem variar de $15 \%$ a $97 \%$, dependendo do cultivar plantado, da época de plantio e da composição e densidade de plantas daninhas. A presença de duas plantas por metro de Solanum sarrachoides reduz a produtividade do feijoeiro em 13\%, em média (Blackshaw, 1991).
A utilização de herbicidas na cultura do feijão vem aumentando ao longo dos anos, tendo como principais razões a alta eficiência no controle de plantas daninhas, o alto rendimento operacional e a redução de custos, em comparação com os métodos de controle mecânico e manual (Procópio et al., 2001). Todavia, sintomas de toxicidade nas plantas de feijão são comumente constatados após a aplicação de herbicidas. Vários fatores podem estar relacionados com esse fato, como: tipo de solo, precipitações, manejo da irrigação, temperatura e cultivar utilizado (Fuentes et al., 1984; Viger et al., 1991; Kunkel et al., 1996). Fuentes et al. (1984) observaram diferenças na sensibilidade de cultivares de feijão ao herbicida linuron. Procópio et al. (2003) verificaram que os cultivares de feijão Carioca e Vermelho 2157 foram mais sensiveis ao herbicida S-metolachlor que os cultivares Pérola e Jalo Precoce.

Apesar da crescente adoção do controle químico de plantas daninhas nas lavouras de feijão, a disponibilidade atual de herbicidas registrados no Brasil para aplicação após a emergência da cultura, principalmente de latifolicidas, é pequena (apenas quatro princípios ativos), quando comparada às opções para a cultura da soja (16 princípios ativos para controle de folhas largas). Esse pequeno número de latifolicidas disponiveis para aplicação na cultura do feijão gera um encarecimento nos custos do controle químico (baixa oferta de produtos e, consequentemente, pequena concorrência entre as empresas distribuidoras das moléculas) e o controle ineficiente de determinadas espécies invasoras pelos poucos herbicidas registrados. Os herbicidas chlorimuron-ethyl, imazethapyr e cloransulam-methyl são produtos registrados 
para uso na cultura da soja, sobretudo em aplicações de pós-emergência, controlando grande número de espécies de plantas daninhas latifoliadas (Kent et al., 1991; Barros et al., 2000; Barnes \& Oliver, 2004). Apesar de o mercado de herbicidas na cultura do feijão no Brasil não ser um dos mais atraentes, não estimulando a pesquisa e o desenvolvimento de outras moléculas pelas empresas fabricantes de defensivos agrícolas, é obrigação dos pesquisadores tentar encontrar novas opções de herbicidas para uso nessa cultura.

O objetivo deste trabalho foi avaliar a seletividade dos herbicidas chlorimuron-ethyl, imazethapyr e cloransulam-methyl, aplicados em associação com o herbicida fomesafen, a dez cultivares de feijão, bem como o potencial de utilização deles na cultura.

\section{MATERIAL E MÉTODOS}

O experimento foi conduzido na fazenda experimental da Fesurv - Universidade de Rio Verde, localizada no município de Rio VerdeGO, cujas coordenadas geográficas são: latitude de $17^{\circ} 47^{\prime} 24^{\prime \prime} \mathrm{S}$, longitude de 5056’31" W e altitude de 698 metros. O solo da área experimental é classificado como Latossolo Vermelho distroférrico. O período de condução do experimento foi de 14/12/2005 a 20/3/2006.

O delineamento experimental utilizado foi de blocos ao acaso, em parcelas subdivididas, sendo o fator da parcela principal os tratamentos com herbicidas [fomesafen (225 $\left.\mathrm{g} \mathrm{ha}^{-1}\right)$; fomesafen (225 $\left.\mathrm{g} \mathrm{ha}^{-1}\right)+$ chlorimuron-ethyl $\left(7,5 \mathrm{~g} \mathrm{ha}^{-1}\right)$; fomesafen $\left(225 \mathrm{~g} \mathrm{ha}^{-1}\right)+$ imazethapyr (50 $\left.\mathrm{g} \mathrm{ha}^{-1}\right)$; fomesafen $\left(225 \mathrm{~g} \mathrm{ha}^{-1}\right)$ + cloransulam-methyl $\left(30,24 \mathrm{~g} \mathrm{ha}^{-1}\right)$; e testemunha sem herbicida], e o da subparcela, os cultivares de feijão (Tabela 1 ). Foram realizadas três repetições. As dimensões da parcela experimental foram de $4 \mathrm{~m}$ de comprimento por $2 \mathrm{~m}$ de largura (quatro linhas de feijão), totalizando $8 \mathrm{~m}^{2}$, sendo a área útil utilizada nas avaliações de $3 \mathrm{~m}$ por $1 \mathrm{~m}$ (duas linhas centrais de feijão).

Utilizou-se o sistema de plantio direto, sendo realizadas duas dessecações de manejo com glyphosate ( $1.440 \mathrm{~g}$ de equivalente ácido $\mathrm{ha}^{-1}$ ): a primeira 20 dias antes do plantio e a segunda três dias antes do plantio. A semeadura do feijão foi realizada manualmente em 14/12/2005, depositando-se dez sementes por metro. O espaçamento utilizado entre linhas foi de $0,50 \mathrm{~m}$. A adubação de plantio constou da aplicação de $400 \mathrm{~kg}^{\mathrm{h}} \mathrm{a}^{-1}$ da fórmula N-P-K 5-25-15. Foi feita uma adubação de cobertura 30 dias após a emergência da cultura, com $200 \mathrm{~kg} \mathrm{ha}^{-1}$ de sulfato de amônio. Todas as parcelas experimentais foram mantidas capinadas, para que não houvesse nenhum tipo de interferência das plantas daninhas nos tratamentos.

Dezenove dias após a emergência das plantas de feijão, foi realizada a aplicação dos tratamentos herbicidas, utilizando-se um pulverizador costal com pressurização por $\mathrm{CO}_{2}$, com quatro pontas de pulverização do tipo TT 110-02. Foi aplicado volume de calda equivalente a $200 \mathrm{~L} \mathrm{ha}^{-1}$. O horário da aplicação foi das 8 às 9h10, e as condições climáticas desse periodo foram: $\mathrm{T}$ mín. $=23,2^{\circ}$; $\mathrm{T}$ média $=24,1^{\circ} ; \mathrm{T}$ máx. $=24,8^{\circ} ; \mathrm{UR}$ mín. $=77 \%$; UR média $=80 \%$; UR máx. $=85 \%$; velocidade do vento min. $=3 \mathrm{~km} \mathrm{~h}^{-1}$; velocidade do vento média $=7 \mathrm{~km} \mathrm{~h}^{-1}$; e velocidade do vento máx. $=9 \mathrm{~km} \mathrm{~h}^{-1}$.

Foram realizadas três avaliações visuais dos sintomas de toxicidade, provocados pelos tratamentos herbicidas, nas plantas de feijão, utilizando-se escala percentual de 0 (zero) a $100 \%$, em que zero representa ausência de sintomas e $100 \%$ morte de todas as plantas, aos 3, 10 e 28 dias após a aplicação (DAA). Aos 16 DAA, realizou-se uma avaliação da altura das plantas de feijão, tendo como referência o meristema apical; também nesta data, coletou-se a parte aérea de seis plantas ao acaso por parcela, as quais foram secas em

Tabela 1 - Cultivares de feijão utilizados no experimento. Rio Verde-GO, 2005/2006

\begin{tabular}{|l|l|l|}
\hline \multicolumn{1}{|c|}{ Cultivar } & Grupo comercial & Porte da planta \\
\hline 1. BRS Grafite & preto & semiereto \\
\hline 2. BRS Horizonte & carioca & ereto \\
\hline 3. Pérola & carioca & semiereto \\
\hline 4. BRS Pitanga & roxinho & ereto \\
\hline 5. BRS Pontal & carioca & semiprostrado \\
\hline 6. BRS Requinte & carioca & semiprostrado \\
\hline 7. BRS Supremo & preto & ereto \\
\hline 8. BRS Timbó & roxinho & semiereto \\
\hline 9. BRS Valente & preto & ereto \\
\hline 10. BRS Vereda & rosinha & semiereto \\
\hline
\end{tabular}

Planta Daninha, Viçosa-MG, v. 27, n. 2, p. 327-336, 2009 
estufa de circulação forçada de ar regulada para $65^{\circ} \mathrm{C} \pm 3^{\circ} \mathrm{C}$, até atingirem massa constante. Posteriormente, os feijoeiros foram pesados em balança analítica, para determinação da massa da parte aérea seca. Após obtenção da altura e da massa da parte aérea de plantas secas, calculou-se a porcentagem de redução dessas variáveis causada pela ação dos tratamentos herbicidas, tendo como base os dados da testemunha que não recebeu herbicida. Determinou-se o ciclo médio de maturação fisiológica das plantas de feijão, ou seja, a data em que as sementes do feijoeiro se encontravam, em média, com $30 \%$ de água. Conforme as parcelas iam atingindo a maturação fisiológica, procedia-se à colheita manual, avaliando a produtividade de grãos e uniformizando o teor de água dos grãos para 14\%. Também, a fim de avaliar a ação dos tratamentos herbicidas sobre os cultivares, os dados de produtividade foram transformados em porcentagem de redução, a partir dos dados coletados da testemunha sem herbicida.

Para os testes estatísticos, incluindo-se a análise de variância, os dados sofreram transformação de escala para $(x+0,5)^{1 / 2}$. As médias das variáveis significativas pelo teste $\mathrm{F}$ foram agrupadas pelo critério de Scott Knott a 5\% de probabilidade.

\section{RESULTADOS E DISCUSSÃO}

Todos os tratamentos herbicidas causaram fitotoxicidade superior à do tratamento testemunha, aos três dias após aplicação (DAA), em todos os cultivares de feijão. Os tratamentos herbicidas fomesafen $\left(225,00 \mathrm{~g} \mathrm{ha}^{-1}\right)$ + chlorimuron-ethyl $\left(7,50 \mathrm{~g} \mathrm{ha}^{-1}\right)$ e fomesafen $\left(225,00 \mathrm{~g} \mathrm{ha}^{-1}\right)+$ cloransulam-methyl $\left(30,24 \mathrm{~g} \mathrm{ha}^{-1}\right)$ foram os mais tóxicos a todos os cultivares de feijão; contudo, no caso do cv. Pérola, eles não diferiram do tratamento fomesafen + imazethapyr (Tabela 2). Aos 3 DAA, a aplicação de fomesafen + imazethapyr ocasionou níveis de intoxicação aos cultivares BRS Grafite, BRS Horizonte, BRS Pitanga, BRS Requinte, BRS Supremo, BRS Timbó, BRS Valente e BRS Vereda semelhantes ao promovido pela aplicação do tratamento padrão para controle de folhas largas no feijoeiro (fomesafen). Os cultivares Pérola e BRS Pontal demonstraram maior suscetibilidade à adição de imazethapyr ao fomesafen; e
BRS Horizonte, BRS Requinte, BRS Supremo, BRS Valente e BRS Vereda apresentaram maior tolerância a essa combinação (Tabela 2). O cultivar BRS Timbó foi mais sensível à aplicação dos tratamentos fomesafen e fomesafen + imazethapyr. Os cultivares BRS Horizonte, Pérola, BRS Requinte e BRS Valente foram mais tolerantes à mistura do chlorimuron-ethyl ao fomesafen. Todos os cultivares apresentaram o mesmo nivel de sintomas após a aplicação de fomesafen + cloransulam-methyl (Tabela 2).

A intensidade dos sintomas de injúrias acarretados pela aplicação dos herbicidas aumentou aos 10 DAA, e novamente todos os tratamentos promoveram toxicidade a todos os cultivares de feijão, em relação à testemunha sem herbicida (Tabela 2). Maior nivel de intoxicação aos cultivares de feijão foi observado com a aplicação de fomesafen + chlorimuronethyl, o qual variou de 52,7\% (Pérola) a 76,7\% (BRS Timbó). Todavia, quanto aos cultivares BRS Horizonte e Pérola, os sintomas provocados por esse tratamento não diferiram do tratamento fomesafen + cloransulam-methyl. Com exceção dos cultivares BRS Horizonte e BRS Vereda, a adição de imazethapyr ao fomesafen, aos 10 DAA, incrementou a toxicidade a todos os cultivares de feijão (Tabela 2). Também, constatou-se que a adição de cloransulam-methyl ao fomesafen provoca maiores injúrias ao feijão do que quando se associa o herbicida imazethapyr. Apenas o cultivar BRS Grafite respondeu semelhantemente à adição desses dois herbicidas utilizados na cultura da soja. Mesmo sendo o fomesafen um herbicida registrado para uso na cultura do feijão, os cultivares apresentamlhe tolerância variável: BRS Grafite, Pérola, BRS Pitanga, BRS Valente e BRS Vereda foram os mais suscetiveis. Wilson (2005) observou injúrias em plantas de feijão aos 7 DAA do fomesafen, porém esses sintomas foram temporários, com plena recuperação da cultura. Todas os cultivares apresentaram sintomas de intoxicação relevantes com a associação do chlorimuron-ethyl ao fomesafen, mas BRS Horizonte, Pérola, BRS Pontal e BRS Requinte mostraram maior tolerância em relação aos demais (Tabela 2). Esses cultivares, com exceção do Pérola, também mostraram menor sensibilidade quando o herbicida adicionado ao fomesafen foi o imazethapyr. Não se constatou 
Tabela 2 - Toxicidade em cultivares de feijão após a aplicação dos tratamentos herbicidas. Rio Verde-GO, 2005/2006

\begin{tabular}{|c|c|c|c|c|c|c|}
\hline \multirow[b]{2}{*}{ Cultivar } & \multicolumn{5}{|c|}{ Tratamento herbicida } & \multirow[b]{2}{*}{ Média } \\
\hline & $\begin{array}{c}\text { Fomesafen } \\
\left(225,00 \mathrm{~g} \mathrm{ha}^{-1}\right)\end{array}$ & $\begin{array}{c}\text { Fomesafen } \\
\left(225,00 \mathrm{~g} \mathrm{ha}^{-1}\right)+ \\
\text { chlorimuron-ethyl } \\
\left(7,50 \mathrm{~g} \mathrm{ha}^{-1}\right)\end{array}$ & $\begin{array}{c}\text { Fomesafen } \\
\left(225,00 \mathrm{~g} \mathrm{ha}^{-1}\right)+ \\
\text { imazethapyr } \\
\left(50,00 \mathrm{~g} \mathrm{ha}^{-1}\right)\end{array}$ & $\begin{array}{c}\text { Fomesafen } \\
\left(225,00 \mathrm{~g} \mathrm{ha}^{-1}\right)+ \\
\text { cloransulam-methyl } \\
\left(30,24 \mathrm{~g} \mathrm{ha}^{-1}\right)\end{array}$ & $\begin{array}{l}\text { Testemunha } \\
\text { sem herbicida }\end{array}$ & \\
\hline & \multicolumn{6}{|c|}{ Fitotoxicidade $(\%)$ aos $3 \mathrm{DAA}^{*}$} \\
\hline BRS Grafite & $5,3 \mathrm{Bb}$ & $12,3 \mathrm{Aa}$ & $6,0 \mathrm{Bb}$ & $12,3 \mathrm{Aa}$ & $0,0 \mathrm{Ac}$ & 7,2 \\
\hline BRS Horizonte & $3,0 \mathrm{Bb}$ & $10,7 \mathrm{Ba}$ & $4,7 \mathrm{Cb}$ & $11,7 \mathrm{Aa}$ & $0,0 \mathrm{Ac}$ & 6,0 \\
\hline Pérola & $3,0 \mathrm{Bb}$ & $10,0 \mathrm{Ba}$ & $6,0 \mathrm{Ba}$ & $8,7 \mathrm{Aa}$ & $0,0 \mathrm{Ac}$ & 5,5 \\
\hline BRS Pitanga & $5,0 \mathrm{Bb}$ & $13,3 \mathrm{Aa}$ & $7,0 \mathrm{Bb}$ & $11,0 \mathrm{Aa}$ & $0,0 \mathrm{Ac}$ & 7,3 \\
\hline BRS Pontal & $3,0 \mathrm{Bc}$ & $12,0 \mathrm{Aa}$ & $7,0 \mathrm{Bb}$ & $10,0 \mathrm{Aa}$ & $0,0 \mathrm{Ad}$ & 6,4 \\
\hline BRS Requinte & $3,3 \mathrm{Bb}$ & $7,3 \mathrm{Ba}$ & $3,7 \mathrm{Cb}$ & $6,7 \mathrm{Aa}$ & $0,0 \mathrm{Ac}$ & 4,2 \\
\hline BRS Supremo & $3,7 \mathrm{Bb}$ & $11,3 \mathrm{Aa}$ & $4,3 \mathrm{Cb}$ & $11,7 \mathrm{Aa}$ & $0,0 \mathrm{Ac}$ & 6,2 \\
\hline BRS Timbó & $9,0 \mathrm{Ab}$ & $16,7 \mathrm{Aa}$ & $11,0 \mathrm{Ab}$ & $14,0 \mathrm{Aa}$ & $0,0 \mathrm{Ac}$ & 10,1 \\
\hline BRS Valente & $4,7 \mathrm{Bb}$ & $9,3 \mathrm{Ba}$ & $3,0 \mathrm{Cb}$ & $10,7 \mathrm{Aa}$ & $0,0 \mathrm{Ac}$ & 5,5 \\
\hline BRS Vereda & $3,7 \mathrm{Bb}$ & $11,7 \mathrm{Aa}$ & $3,0 \mathrm{Cb}$ & $10,0 \mathrm{Aa}$ & $0,0 \mathrm{Ac}$ & 5,7 \\
\hline \multirow[t]{2}{*}{ Média } & 4,4 & 11,5 & 5,6 & 10,7 & 0,0 & 6,4 \\
\hline & \multicolumn{6}{|c|}{ Fitotoxicidade (\%) aos 10 DAA } \\
\hline BRS Grafite & $15,0 \mathrm{Ac}$ & $66,7 \mathrm{Aa}$ & $29,0 \mathrm{Ab}$ & $39,3 \mathrm{Ab}$ & $0,0 \mathrm{Ad}$ & 30,0 \\
\hline BRS Horizonte & $10,7 \mathrm{Bb}$ & $55,7 \mathrm{Ba}$ & $13,0 \mathrm{Bb}$ & $46,3 \mathrm{Aa}$ & $0,0 \mathrm{Ac}$ & 25,1 \\
\hline Pérola & $12,7 \mathrm{Ac}$ & $52,7 \mathrm{Ba}$ & $26,7 \mathrm{Ab}$ & $46,0 \mathrm{Aa}$ & $0,0 \mathrm{Ad}$ & 27,6 \\
\hline BRS Pitanga & $12,3 \mathrm{Ad}$ & $65,7 \mathrm{Aa}$ & $28,3 \mathrm{Ac}$ & $39,3 \mathrm{Ab}$ & $0,0 \mathrm{Ae}$ & 29,1 \\
\hline BRS Pontal & $9,7 \mathrm{Bd}$ & $56,3 \mathrm{Ba}$ & $20,7 \mathrm{Bc}$ & $35,0 \mathrm{Ab}$ & $0,0 \mathrm{Ae}$ & 24,3 \\
\hline BRS Requinte & $8,3 \mathrm{Bd}$ & $54,7 \mathrm{Ba}$ & $18,3 \mathrm{Bc}$ & $39,3 \mathrm{Ab}$ & $0,0 \mathrm{Ae}$ & 24,1 \\
\hline BRS Supremo & $9,3 \mathrm{Bd}$ & $67,3 \mathrm{Aa}$ & $26,7 \mathrm{Ac}$ & $43,0 \mathrm{Ab}$ & $0,0 \mathrm{Ae}$ & 29,3 \\
\hline BRS Timbó & $8,0 \mathrm{Bd}$ & $76,7 \mathrm{Aa}$ & $25,7 \mathrm{Ac}$ & $37,3 \mathrm{Ab}$ & $0,0 \mathrm{Ae}$ & 29,5 \\
\hline BRS Valente & $15,3 \mathrm{Ad}$ & $61,7 \mathrm{Aa}$ & $25,0 \mathrm{Ac}$ & $45,7 \mathrm{Ab}$ & $0,0 \mathrm{Ae}$ & 29,5 \\
\hline BRS Vereda & $16,0 \mathrm{Ac}$ & $64,7 \mathrm{Aa}$ & $23,3 \mathrm{Ac}$ & $44,7 \mathrm{Ab}$ & $0,0 \mathrm{Ad}$ & 29,7 \\
\hline \multirow[t]{2}{*}{ Média } & 11,7 & 62,2 & 23,7 & 41,6 & 0,0 & 27,8 \\
\hline & \multicolumn{6}{|c|}{ Fitotoxicidade (\%) aos $28 \mathrm{DAA}$} \\
\hline BRS Grafite & $4,3 \mathrm{Ab}$ & $35,0 \mathrm{Aa}$ & $9,0 \mathrm{Ab}$ & $10,0 \mathrm{Ab}$ & $0,0 \mathrm{Ac}$ & 11,7 \\
\hline BRS Horizonte & $5,0 \mathrm{Ab}$ & $7,7 \mathrm{Ca}$ & $1,0 \mathrm{Ab}$ & $8,7 \mathrm{Aa}$ & $0,0 \mathrm{Ab}$ & 4,5 \\
\hline Pérola & $3,7 \mathrm{Ab}$ & $15,3 \mathrm{Ca}$ & $12,3 \mathrm{Aa}$ & $8,3 \mathrm{Aa}$ & $0,0 \mathrm{Ab}$ & 7,9 \\
\hline BRS Pitanga & $3,3 \mathrm{Ab}$ & $19,3 \mathrm{Ba}$ & $6,7 \mathrm{Ab}$ & $5,7 \mathrm{Ab}$ & $0,0 \mathrm{Aa}$ & 7,0 \\
\hline BRS Pontal & $4,7 \mathrm{Ab}$ & $11,7 \mathrm{Ca}$ & $13,0 \mathrm{Aa}$ & $8,3 \mathrm{Aa}$ & $0,0 \mathrm{Ab}$ & 7,5 \\
\hline BRS Requinte & $1,7 \mathrm{Ab}$ & $5,7 \mathrm{Cb}$ & $14,3 \mathrm{Aa}$ & $9,3 \mathrm{Aa}$ & $0,0 \mathrm{Ab}$ & 6,2 \\
\hline BRS Supremo & $1,0 \mathrm{Ac}$ & $23,7 \mathrm{Ba}$ & $9,3 \mathrm{Ab}$ & $7,7 \mathrm{Ab}$ & $0,0 \mathrm{Ac}$ & 8,3 \\
\hline BRS Timbó & $5,0 \mathrm{Ac}$ & $50,0 \mathrm{Aa}$ & $15,0 \mathrm{Ab}$ & $12,0 \mathrm{Ab}$ & $0,0 \mathrm{Ac}$ & 16,4 \\
\hline BRS Valente & $0,0 \mathrm{Ac}$ & $26,7 \mathrm{Ba}$ & $6,3 \mathrm{Ab}$ & $8,7 \mathrm{Ab}$ & $0,0 \mathrm{Ac}$ & 8,3 \\
\hline BRS Vereda & $4,0 \mathrm{Ab}$ & $22,3 \mathrm{Ba}$ & $13,7 \mathrm{Aa}$ & $10,7 \mathrm{Aa}$ & $0,0 \mathrm{Ab}$ & 10,1 \\
\hline Média & 3,3 & 21,7 & 10,1 & 8,9 & 0,0 & 8,8 \\
\hline
\end{tabular}

Médias seguidas pelas mesmas letras, minúsculas na linha (horizontal) e maiúsculas na coluna (vertical), não diferem estatisticamente entre si pelo teste de Scott Knott a 5\% de probabilidade. * DAA = dias após a aplicação.

diferença entre os cultivares de feijão quanto à tolerância à mistura de cloransulam-methyl e fomesafen, aos 10 DAA. A exteriorização dos sintomas de fitotoxicidade foi muito semelhante entre os tratamentos com os herbicidas inibidores de ALS: amarelecimento das folhas com necrose das nervuras, principalmente na face abaxial, diferenciando-se apenas quanto ao grau da injúria.
Aos 28 DAA, observou-se, em geral, redução da fitotoxicidade promovida pelos tratamentos químicos em todos os cultivares de feijão, evidenciando a capacidade de recuperação desses materiais mesmo com a utilização de herbicidas não registrados para o feijoeiro. Com exceção do cultivar BRS Requinte, que apresentou maior sensibilidade aos tratamentos fomesafen + imazethapyr e 
fomesafen + cloransulam-methyl, os demais continuaram mais intoxicados com a utilização de fomesafen + chlorimuron-ethyl. Nessa última avaliação de fitotoxicidade, as injúrias causadas com a adição de imazethapyr ou de cloransulam-methyl ao fomesafen sobre os cultivares BRS Grafite e BRS Pitanga não diferiram das ocasionadas pela aplicação isolada de fomesafen - comportamento esse semelhante ao do cultivar BRS Horizonte em relação à adição de imazethapyr e para o cultivar BRS Requinte com a inclusão de chlorimuron-ethyl. Quanto aos demais cultivares, a associação de herbicidas não registrados para uso nessa cultura promoveu maior intoxicação às plantas de feijão. Vangessel et al. (2000) constataram após a aplicação em pós-emergência do herbicida cloransulammethyl, na dose de $20 \mathrm{~g} \mathrm{ha}^{-1}$, em um cultivar norte-americano de Phaseolus lunatus, intoxicação variando de 3 a 13\%, conforme a localidade do experimento. Todos os cultivares de feijão apresentaram o mesmo grau de suscetibilidade aos tratamentos herbicidas fomesafen, fomesafen + imazethapyr e fomesafen + cloransulam-methyl. Contudo, quando se aplicou a mistura de fomesafen + chlorimuron-ethyl, BRS Timbó e BRS Grafite foram os mais suscetiveis, apresentando, aos 28 DAA, 50 e 35\% de toxicidade, respectivamente. BRS Horizonte, Pérola, BRS Pontal e BRS Requinte foram os mais tolerantes (Tabela 2).

A aplicação de fomesafen + chlorimuronethyl provocou a maior redução da altura das plantas dos cultivares de feijão, apenas não diferindo da redução acarretada pelo tratamento fomesafen + cloransulam-methyl nos cultivares BRS Grafite e Pérola (Tabela 3). A associação de imazethapyr ao fomesafen acentuou apenas a redução do porte da planta do cultivar BRS Pitanga. No entanto, quando o herbicida adicionado ao fomesafen foi o cloransulam-methyl, observou-se maior redução na altura das plantas dos cultivares BRS Grafite, Pérola, BRS Pitanga, BRS Timbó e BRS Valente. Wilson (2005) constatou que a mistura em tanque do fomesafen com o imazamox causou maior injúria à cultura do feijão do que a combinação de fomesafen com o herbicida bentazon. A utilização isolada do fomesafen apenas proporcionou redução da altura das plantas do cultivar BRS Requinte (Tabela 3). Não houve diferença na resposta do porte dos cultivares quando foram aplicados os tratamentos fomesafen, fomesafen + chlorimuron-ethyl e fomesafen + imazethapyr. Entretanto, BRS Grafite, Pérola, BRS Requinte, BRS Timbó e BRS Valente foram os que apresentaram as maiores reduções na altura quando se pulverizaram as plantas com fomesafen

Tabela 3 - Redução na altura de plantas de cultivares de feijão após aplicação dos tratamentos herbicidas. Rio Verde-GO, 2005/2006

\begin{tabular}{|c|c|c|c|c|c|c|}
\hline \multirow{3}{*}{ Cultivar } & \multicolumn{5}{|c|}{ Tratamento herbicida } & \multirow[b]{2}{*}{ Média } \\
\hline & $\begin{array}{c}\text { Fomesafen } \\
\left(225,00 \mathrm{~g} \mathrm{ha}^{-1}\right)\end{array}$ & $\begin{array}{c}\text { Fomesafen } \\
\left(225,00 \mathrm{~g} \mathrm{ha}^{-1}\right)+ \\
\text { chlorimuron-ethyl } \\
\left(7,50 \mathrm{~g} \mathrm{ha}^{-1}\right)\end{array}$ & $\begin{array}{c}\text { Fomesafen } \\
\left(225,00 \mathrm{~g} \mathrm{ha}^{-1}\right)+ \\
\text { imazethapyr } \\
\left(50,00 \mathrm{~g} \mathrm{ha}^{-1}\right)\end{array}$ & $\begin{array}{c}\text { Fomesafen } \\
\left(225,00 \mathrm{~g} \mathrm{ha}^{-1}\right)+ \\
\text { cloransulam-methyl } \\
\left(30,24 \mathrm{~g} \mathrm{ha}^{-1}\right)\end{array}$ & $\begin{array}{c}\text { Testemunha } \\
\text { sem herbicida }\end{array}$ & \\
\hline & \multicolumn{6}{|c|}{ Porcentagem de redução da altura de plantas aos 16 DAA* } \\
\hline BRS Grafite & $4,8 \mathrm{Ab}$ & $36,2 \mathrm{Aa}$ & $0,0 \mathrm{Ab}$ & $25,4 \mathrm{Aa}$ & $0,0 \mathrm{Ab}$ & 13,3 \\
\hline BRS Horizonte & $0,0 \mathrm{Ab}$ & $33,8 \mathrm{Aa}$ & $0,0 \mathrm{Ab}$ & $0,8 \mathrm{Bb}$ & $0,0 \mathrm{Ab}$ & 6,9 \\
\hline Pérola & $1,8 \mathrm{Ab}$ & $33,4 \mathrm{Aa}$ & $8,5 \mathrm{Ab}$ & $19,9 \mathrm{Aa}$ & $0,0 \mathrm{Ab}$ & 12,7 \\
\hline BRS Pitanga & $0,6 \mathrm{Ac}$ & $37,5 \mathrm{Aa}$ & $8,5 \mathrm{Ab}$ & $7,0 \mathrm{Bb}$ & $0,0 \mathrm{Ac}$ & 10,7 \\
\hline BRS Pontal & $1,1 \mathrm{Ab}$ & $40,7 \mathrm{Aa}$ & $0,0 \mathrm{Ab}$ & $0,0 \mathrm{Bb}$ & $0,0 \mathrm{Ab}$ & 8,4 \\
\hline BRS Requinte & $8,8 \mathrm{Ab}$ & $55,0 \mathrm{Aa}$ & $5,7 \mathrm{Ab}$ & $12,1 \mathrm{Ab}$ & $0,0 \mathrm{Ac}$ & 16,3 \\
\hline BRS Supremo & $0,0 \mathrm{Ab}$ & $25,8 \mathrm{Aa}$ & $1,7 \mathrm{Ab}$ & $5,9 \mathrm{Bb}$ & $0,0 \mathrm{Ab}$ & 6,7 \\
\hline BRS Timbó & $0,0 \mathrm{Ac}$ & $34,3 \mathrm{Aa}$ & $3,8 \mathrm{Ac}$ & $14,7 \mathrm{Ab}$ & $0,0 \mathrm{Ac}$ & 10,6 \\
\hline BRS Valente & $2,6 \mathrm{Ac}$ & $36,7 \mathrm{Aa}$ & $1,5 \mathrm{Ac}$ & $16,7 \mathrm{Ab}$ & $0,0 \mathrm{Ac}$ & 11,5 \\
\hline BRS Vereda & $1,3 \mathrm{Ab}$ & $21,5 \mathrm{Aa}$ & $6,9 \mathrm{Ab}$ & $9,4 \mathrm{Bb}$ & $0,0 \mathrm{Ab}$ & 7,8 \\
\hline Média & 2,1 & 35,5 & 3,7 & 11,2 & 0,0 & 10,4 \\
\hline
\end{tabular}

Médias seguidas pelas mesmas letras, minúsculas na linha (horizontal) e maiúsculas na coluna (vertical), não diferem estatisticamente entre si pelo teste de Scott Knott a 5\% de probabilidade. * DAA = dias após a aplicação. 
+ cloransulam-methyl (Tabela 3). Sikkema et al. (2006) constataram que a aplicação de imazethapyr em pré-plantio incorporado (PPI) em área cultivada com feijão reduziu a altura de plantas, a massa da parte aérea seca e a produtividade de grãos em 8,18 e $12 \%$ na dose de $75 \mathrm{~g} \mathrm{ha}^{-1}$ e 19, 38 e $27 \%$ quando a dose do herbicida foi de $150 \mathrm{~g} \mathrm{ha}^{-1}$, respectivamente.

O herbicida adicionado ao fomesafen que mais provocou redução da massa da parte aérea seca dos cultivares de feijão foi o chlorimuronethyl, não diferindo do emprego do cloransulammethyl apenas nos cultivares BRS Grafite, Pérola e BRS Valente (Tabela 4). Os tratamentos fomesafen e fomesafen + imazethapyr não ocasionaram redução significativa na massa da parte aérea seca dos cultivares. Contudo, a aplicação de fomesafen + cloransulammethyl diminuiu o acúmulo de fitomassa dos cultivares BRS Grafite, Pérola, BRS Requinte, BRS Supremo e BRS Valente. Os cultivares de feijão apresentaram acúmulo de massa na parte aérea seca semelhante após a aplicação dos tratamentos fomesafen e fomesafen + imazethapyr. Apesar de promover significativa redução na massa da parte aérea seca dos cultivares, BRS Grafite, BRS Horizonte, BRS Pitanga, BRS Requinte, BRS Timbó e BRS Valente foram os mais afetados pela associação do chlorimuron-ethyl ao fomesafen. Nesses casos, houve reduções de 40,7 a 57,6\%. BRS Grafite, Pérola, BRS Requinte e BRS Valente foram os cultivares mais sensiveis ao cloransulam-methyl quanto à redução do acúmulo de massa na parte aérea seca (Tabela 4). Vangessel et al. (2000) não constataram diferença de sensibilidade entre oito cultivares comerciais de Phaseolus lunatus ao herbicida cloransulam-methyl aplicado em pós-emergência.

Não houve interação entre tratamentos herbicidas e cultivares de feijão quanto à duração do ciclo de maturação dos cultivares. Ocorreram apenas efeitos isolados de cada variável. A aplicação de fomesafen + chlorimuron-ethyl provocou atraso na maturação dos cultivares, em média, de quase cinco dias (Tabela 5). Os outros tratamentos herbicidas não tiveram influência na maturação dos cultivares. Sikkema et al. (2004) verificaram que a aplicação em pós-emergência dos herbicidas cloransulam-methyl e chlorimuron-ethyl em dois cultivares de feijão-branco (Phaseolus vulgaris) resultou em aumento no teor de água dos grãos acima de 3,9 e 15,5\%, respectivamente. Os cultivares apresentaram variações quanto à duração do ciclo de maturação: BRS Horizonte foi o mais precoce, com ciclo

Tabela 4 - Redução na massa da parte aérea de plantas secas de cultivares de feijão após aplicação de diferentes tratamentos herbicidas. Rio Verde-GO, 2005/2006

\begin{tabular}{|c|c|c|c|c|c|c|}
\hline \multirow{3}{*}{ Cultivar } & \multicolumn{5}{|c|}{ Tratamento herbicida } & \multirow[b]{2}{*}{ Média } \\
\hline & $\begin{array}{c}\text { Fomesafen } \\
\left(225,00 \mathrm{~g} \mathrm{ha}^{-1}\right)\end{array}$ & $\begin{array}{c}\text { Fomesafen } \\
\left(225,00 \mathrm{~g} \mathrm{ha}^{-1}\right)+ \\
\text { chlorimuron-ethyl } \\
\left(7,50 \mathrm{~g} \mathrm{ha}^{-1}\right)\end{array}$ & $\begin{array}{c}\text { Fomesafen } \\
\left(225,00 \mathrm{~g} \mathrm{ha}^{-1}\right)+ \\
\text { imazethapyr } \\
\left(50,00 \mathrm{~g} \mathrm{ha}^{-1}\right)\end{array}$ & $\begin{array}{c}\text { Fomesafen } \\
\left(225,00 \mathrm{~g} \mathrm{ha}^{-1}\right)+ \\
\text { cloransulam-methyl } \\
\left(30,24 \mathrm{~g} \mathrm{ha}^{-1}\right)\end{array}$ & $\begin{array}{c}\text { Testemunha } \\
\text { sem herbicida }\end{array}$ & \\
\hline & \multicolumn{6}{|c|}{ Porcentagem de redução de massa seca da parte aérea aos 16 DAA* } \\
\hline BRS Grafite & $14,4 \mathrm{Ab}$ & $57,6 \mathrm{Aa}$ & $0,0 \mathrm{Ab}$ & $34,4 \mathrm{Aa}$ & $0,0 \mathrm{Ab}$ & 21,3 \\
\hline BRS Horizonte & $3,7 \mathrm{Ab}$ & $42,2 \mathrm{Aa}$ & $0,0 \mathrm{Ab}$ & $0,0 \mathrm{Bb}$ & $0,0 \mathrm{Ab}$ & 9,2 \\
\hline Pérola & $8,6 \mathrm{Ab}$ & $21,3 \mathrm{Ba}$ & $3,2 \mathrm{Ab}$ & $21,3 \mathrm{Aa}$ & $0,0 \mathrm{Ab}$ & 10,9 \\
\hline BRS Pitanga & $0,0 \mathrm{Ab}$ & $56,3 \mathrm{Aa}$ & $17,7 \mathrm{Ab}$ & $6,6 \mathrm{Bb}$ & $0,0 \mathrm{Ab}$ & 16,1 \\
\hline BRS Pontal & $2,2 \mathrm{Ab}$ & $15,6 \mathrm{Ba}$ & $1,4 \mathrm{Ab}$ & $0,0 \mathrm{Bb}$ & $0,0 \mathrm{Ab}$ & 3,8 \\
\hline BRS Requinte & $9,2 \mathrm{Ac}$ & $53,4 \mathrm{Aa}$ & $0,0 \mathrm{Ac}$ & $24,1 \mathrm{Ab}$ & $0,0 \mathrm{Ac}$ & 17,3 \\
\hline BRS Supremo & $0,0 \mathrm{Ac}$ & $31,5 \mathrm{Ba}$ & $5,4 \mathrm{Ac}$ & $11,2 \mathrm{Bb}$ & $0,0 \mathrm{Ac}$ & 9,6 \\
\hline BRS Timbó & $10,9 \mathrm{Ab}$ & $43,8 \mathrm{Aa}$ & $10,6 \mathrm{Ab}$ & $10,2 \mathrm{Bb}$ & $0,0 \mathrm{Ab}$ & 15,1 \\
\hline BRS Valente & $2,5 \mathrm{Ab}$ & $40,7 \mathrm{Aa}$ & $0,0 \mathrm{Ab}$ & $23,0 \mathrm{Aa}$ & $0,0 \mathrm{Ab}$ & 13,2 \\
\hline BRS Vereda & $0,0 \mathrm{Ab}$ & $21,8 \mathrm{Ba}$ & $4,8 \mathrm{Ab}$ & $5,3 \mathrm{Bb}$ & $0,0 \mathrm{Ab}$ & 6,4 \\
\hline Média & 5,2 & 38,4 & 4,3 & 13,6 & 0,0 & 12,8 \\
\hline
\end{tabular}

Médias seguidas pelas mesmas letras, minúsculas na linha (horizontal) e maiúsculas na coluna (vertical), não diferem estatisticamente entre si pelo teste de Scott Knott a $5 \%$ de probabilidade. ${ }^{*}$ DAA $=$ dias após a aplicação. 
médio de 76,3 dias, enquanto BRS Grafite, BRS Timbó e BRS Valente foram os mais tardios, com ciclo médio variando de 84,1 a 85,7 dias (Tabela 5).

A aplicação do herbicida fomesafen reduziu significativamente a produtividade de grãos das variedades BRS Timbó e BRS Vereda (Tabela 6) - fato preocupante, pois se trata de um herbicida registrado para uso na cultura e amplamente empregado no controle em pósemergência inicial de plantas daninhas latifoliadas. A adição do herbicida imazethapyr ao fomesafen promoveu redução na produtividade

Tabela 5 - Dias da emergência à maturação fisiológica de cultivares de feijão após aplicação de diferentes tratamentos herbicidas. Rio Verde-GO, 2005/2006

\begin{tabular}{|c|c|c|c|c|c|c|}
\hline \multirow{3}{*}{ Cultivar } & \multicolumn{5}{|c|}{ Tratamento herbicida } & \multirow[b]{2}{*}{ Média } \\
\hline & $\begin{array}{c}\text { Fomesafen } \\
\left(225,00 \mathrm{~g} \mathrm{ha}^{-1}\right)\end{array}$ & $\begin{array}{c}\text { Fomesafen } \\
\left(225,00 \mathrm{~g} \mathrm{ha}^{-1}\right)+ \\
\text { chlorimuron-ethyl } \\
\left(7,50 \mathrm{~g} \mathrm{ha}^{-1}\right)\end{array}$ & $\begin{array}{c}\text { Fomesafen } \\
\left(225,00 \mathrm{~g} \mathrm{ha}^{-1}\right)+ \\
\text { imazethapyr } \\
\left(50,00 \mathrm{~g} \mathrm{ha}^{-1}\right)\end{array}$ & $\begin{array}{c}\text { Fomesafen } \\
\left(225,00 \mathrm{~g} \mathrm{ha}^{-1}\right)+ \\
\text { cloransulam-methyl } \\
\left(30,24 \mathrm{~g} \mathrm{ha}^{-1}\right)\end{array}$ & $\begin{array}{l}\text { Testemunha } \\
\text { sem herbicida }\end{array}$ & \\
\hline & \multicolumn{6}{|c|}{ Ciclo médio de maturação dos cultivares de feijão (dias) } \\
\hline BRS Grafite & 83,7 & 86,7 & 84,0 & 83,0 & 83,0 & $84,1 \mathrm{~A}$ \\
\hline BRS Horizonte & 75,0 & 82,0 & 75,7 & 75,0 & 73,7 & $76,3 \mathrm{D}$ \\
\hline Pérola & 83,3 & 83,7 & 83,3 & 80,0 & 80,3 & $82,1 \mathrm{~B}$ \\
\hline BRS Pitanga & 79,0 & 83,3 & 77,7 & 80,0 & 78,3 & $79,7 \mathrm{C}$ \\
\hline BRS Pontal & 77,0 & 83,3 & 78,0 & 79,3 & 77,0 & $78,9 \mathrm{C}$ \\
\hline BRS Requinte & 78,0 & 82,3 & 79,0 & 78,3 & 77,0 & $78,9 \mathrm{C}$ \\
\hline BRS Supremo & 81,0 & 85,3 & 82,3 & 83,3 & 81,0 & $82,6 \mathrm{~B}$ \\
\hline BRS Timbó & 83,0 & 87,0 & 84,7 & 85,3 & 82,3 & $84,5 \mathrm{~A}$ \\
\hline BRS Valente & 85,0 & 87,7 & 85,3 & 84,7 & 85,7 & $85,7 \mathrm{~A}$ \\
\hline BRS Vereda & 82,7 & 86,3 & 82,7 & 84,3 & 81,0 & $83,4 \mathrm{~B}$ \\
\hline Média & $80,8 \mathrm{~b}$ & $84,8 \mathrm{a}$ & $81,3 \mathrm{~b}$ & $81,3 \mathrm{~b}$ & $79,9 \mathrm{~b}$ & 81,6 \\
\hline
\end{tabular}

Médias seguidas pelas mesmas letras, minúsculas na linha (horizontal) e maiúsculas na coluna (vertical), não diferem estatisticamente entre si pelo teste de Scott Knott a 5\% de probabilidade.

Tabela 6 - Redução na produtividade de grãos de cultivares de feijão após aplicação de diferentes tratamentos herbicidas. Rio VerdeGO, 2005/2006

\begin{tabular}{|c|c|c|c|c|c|c|}
\hline \multirow{3}{*}{ Cultivar } & \multicolumn{5}{|c|}{ Tratamento herbicida } & \multirow[b]{2}{*}{ Média } \\
\hline & $\begin{array}{c}\text { Fomesafen } \\
\left(225,00 \mathrm{~g} \mathrm{ha}^{-1}\right)\end{array}$ & $\begin{array}{c}\text { Fomesafen } \\
\left(225,00 \mathrm{~g} \mathrm{ha}^{-1}\right)+ \\
\text { chlorimuron-ethyl } \\
\left(7,50 \mathrm{~g} \mathrm{ha}^{-1}\right)\end{array}$ & $\begin{array}{c}\text { Fomesafen } \\
\left(225,00 \mathrm{~g} \mathrm{ha}^{-1}\right)+ \\
\text { imazethapyr } \\
\left(50,00 \mathrm{~g} \mathrm{ha}^{-1}\right)\end{array}$ & $\begin{array}{c}\text { Fomesafen } \\
\left(225,00 \mathrm{~g} \mathrm{ha}^{-1}\right)+ \\
\text { cloransulam-methyl } \\
\left(30,24 \mathrm{~g} \mathrm{ha}^{-1}\right)\end{array}$ & $\begin{array}{l}\text { Testemunha } \\
\text { sem herbicida }\end{array}$ & \\
\hline & \multicolumn{6}{|c|}{ Porcentagem de redução da produtividade de grãos } \\
\hline BRS Grafite & $9,9 \mathrm{Cb}$ & $24,1 \mathrm{Ba}$ & $3,1 \mathrm{Bb}$ & $9,7 \mathrm{Bb}$ & $0,0 \mathrm{Ab}$ & 9,4 \\
\hline BRS Horizonte & $6,0 \mathrm{Cb}$ & $19,9 \mathrm{Ba}$ & $0,0 \mathrm{Bb}$ & $5,8 \mathrm{Bb}$ & $0,0 \mathrm{Aa}$ & 6,3 \\
\hline Pérola & $0,0 \mathrm{Ca}$ & $0,0 \mathrm{Ca}$ & $0,0 \mathrm{Ba}$ & $1,5 \mathrm{Ba}$ & $0,0 \mathrm{Aa}$ & 0,3 \\
\hline BRS Pitanga & $0,0 \mathrm{Ca}$ & $9,5 \mathrm{Ca}$ & $9,8 \mathrm{Aa}$ & $0,9 \mathrm{Ba}$ & $0,0 \mathrm{Aa}$ & 4,0 \\
\hline BRS Pontal & $2,9 \mathrm{Ca}$ & $0,0 \mathrm{Ca}$ & $0,0 \mathrm{Ba}$ & $7,6 \mathrm{Ba}$ & $0,0 \mathrm{Aa}$ & 2,1 \\
\hline BRS Requinte & $3,3 \mathrm{Cb}$ & $30,7 \mathrm{Ba}$ & $7,8 \mathrm{Ab}$ & $28,0 \mathrm{Aa}$ & $0,0 \mathrm{Ab}$ & 14,0 \\
\hline BRS Supremo & $0,6 \mathrm{Cb}$ & $14,9 \mathrm{Ca}$ & $17,6 \mathrm{Aa}$ & $32,3 \mathrm{Aa}$ & $0,0 \mathrm{Ab}$ & 13,1 \\
\hline BRS Timbó & $23,5 \mathrm{Ab}$ & $54,9 \mathrm{Aa}$ & $17,3 \mathrm{Ab}$ & $48,2 \mathrm{Aa}$ & $0,0 \mathrm{Ac}$ & 28,8 \\
\hline BRS Valente & $11,4 \mathrm{Ba}$ & $11,0 \mathrm{Ca}$ & $5,9 \mathrm{Ba}$ & $8,5 \mathrm{Ba}$ & $0,0 \mathrm{Aa}$ & 7,4 \\
\hline BRS Vereda & $15,7 \mathrm{Bb}$ & $39,7 \mathrm{Aa}$ & $16,8 \mathrm{Ab}$ & $46,0 \mathrm{Aa}$ & $0,0 \mathrm{Ac}$ & 23,6 \\
\hline Média & 7,3 & 20,5 & 7,8 & 18,8 & 0,0 & 11,7 \\
\hline
\end{tabular}

Médias seguidas pelas mesmas letras, minúsculas na linha (horizontal) e maiúsculas na coluna (vertical), não diferem estatisticamente entre si pelo teste de Scott Knott a 5\% de probabilidade. 
de grãos dos cultivares BRS Supremo, BRS Timbó e BRS Vereda, mas não alterou a produtividade dos demais, enquanto a adição de cloransulam-methyl, além de reduzir a produtividade desses três cultivares, promoveu também diminuição significativa da produtividade do cultivar BRS Requinte (Tabela 6). Apenas Pérola, BRS Pitanga, BRS Pontal e BRS Valente não tiveram suas produtividades reduzidas pela mistura do herbicida chlorimuronethyl com o fomesafen. A redução na produtividade de grãos observada com o emprego do chlorimuron-ethyl atingiu $54,9 \%$ no cultivar BRS Timbó. Este, juntamente com BRS Vereda (39,7\% de redução na produtividade), teve maior redução da produtividade com a utilização desse herbicida. BRS Timbó foi o cultivar que mais teve diminuição da produtividade com a aplicação isolada do fomesafen (Tabela 6). Machado et al. (2006) verificaram que a produtividade do feijoeiro cultivar Meia Noite não foi afetada pela aplicação da mistura dos herbicidas fomesafen e fluazifop-p-butil nas doses de $100+80$ e $200+160 \mathrm{~g} \mathrm{ha}^{-1}$, respectivamente. Com a aplicação do tratamento fomesafen + imazethapyr, os cultivares BRS Pitanga, BRS Requinte, BRS Supremo, BRS Timbó e BRS Vereda tiveram as maiores reduções de produtividade; o mesmo ocorreu após o emprego do fomesafen + cloransulammethyl, com exceção do cultivar BRS Pitanga. Sikkema et al. (2004) afirmam que os herbicidas chlorimuron-ethyl e cloransulam-methyl, aplicados em pós-emergência, não apresentam seletividade suficiente para serem registrados para uso na cultura do feijão-branco.

Entre os herbicidas ainda não registrados para uso na cultura do feijão avaliados no presente trabalho, imazethapyr mostrou condições de ser utilizado nessa cultura. Soltani et al. (2005) concluíram que o imazethapyr aplicado em PPI apresenta margem de segurança suficiente para seu uso no controle de plantas daninhas na cultura do feijão-adzuki (Vigna angularis). Contudo, experimentos em outras localidades, com diferentes condições climáticas e tipos de solo, envolvendo mais cultivares de feijão de importância econômica, deverão ser realizados a fim de se formar um banco de dados que permita assegurar a seletividade e, consequentemente, fornecer subsídios consistentes para o requerimento de seu registro para uso na cultura do feijão.

\section{LITERATURA CITADA}

BARNES, J. W.; OLIVER, L. R. Cloransulam absorption, translocation, and efficacy on common broadleaf weed species. Weed Sci., v. 52, n. 4, p. 634-641, 2004.

BARROS, A. C. et al. Efeitos de latifolicidas aplicados em pós-emergência sobre algumas características agronômicas de soja cv. Emgopa 316. R. Bras. Herbic., v. 1, n. 2, p. $153-158,2000$.

BLACKSHAW, R. E. Hairy nigthshade (Solanum sarrachoides) interference in dry beans (Phaseolus vulgaris). Weed Sci., v. 39, n. 1, p. 48-53, 1991.

BURNSIDE, O. C. et al. Critical periods for weed control in dry beans (Phaseolus vulgaris). Weed Sci., v. 46, n. 3, p. 301-306, 1998.

COBUCCI, T; FERREIRA, F. A.; SILVA, A. A. Controle de plantas daninhas. In: ARAUJO, R. S. et al. (Eds.). Cultura do feijoeiro comum no Brasil. Piracicaba: POTAFOS, 1996. p. 433-464.

FUENTES, J. R. et al. Tolerância de cultivares de feijão (Phaseolus vulgaris L.) aos herbicidas alachlor e linuron. R. Ceres, v. 31, n. 174, p. 136-145, 1984.

FUSCALDI, K. C.; PRADO, G. R. Análise econômica da cultura do feijão. R. Política Agric., v. 14, n. 1, p. 17-30, 2005 .

KENT, L. M.; WILLS, G. D.; SHAW, D. R. Influence of ammonium sulfate, imazapyr, temperature, and relative humidity on the absorption and translocation of imazethapyr. Weed Sci., v. 39, n. 3, p. 412-416, 1991.

KUNKEL, D. L; BELLINDER, R. R.; STEFFENS, J. C. Safeners reduce corn (Zea mays) chloroacetanilide and dicamba injury under different soil temperatures. Weed Technol., v. 10, n. 1, p. 115-120, 1996.

LUNKES, J. A. Manejo integrado de plantas daninhas na cultura do feijão. In: FANCELLI, A. L.; DOURADO-NETO, D. Tecnologia da produção do feijão irrigado. Piracicaba: ESALQ/USP. Departamento de Agricultura, 1997. p. 9-19.

MACHADO, A. F. L. et al. Misturas de herbicidas no manejo de plantas daninhas na cultura do feijão. Planta Daninha, v. 24, n. 1, p.107-114, 2006.

PROCÓPIO, S. O. et al. Seletividade do S-metolachlor a cultivares de feijão (Phaseolus vulgaris L.). Ci. Agrotecnol., v. 27, n. 1, p. $150-157,2003$.

PROCÓPIO, S. O. et al. Tolerância de cultivares de feijão ao S-metolachlor em diferentes condições de aplicação. Planta Daninha, v. 19, n. 2, p. 263-271, 2001.

Planta Daninha, Viçosa-MG, v. 27, n. 2, p. 327-336, 2009 
SILVA, R. J. S.; VAHL, L. C.; PESKE, S. T. Rendimento de grãos no feijoeiro em função dos teores de fósforo nas sementes. R. Bras. Agroci., v. 9, n. 3, p. 247-250, 2003.

SIKKEMA, P. H. et al. Tolerance of white beans to postemergence broadleaf herbicides. Weed Technol., v. 18, n. 4, p. 893-901, 2004.

SIKKEMA, P. H. et al. Tolerance of otebo bean (Phaseolus vulgaris) to new herbicides in Ontario. Weed Technol., v. 20 , n. 4, p. 862-866, 2006.

SOLTANI, N. et al. Sensitivity of adzuki bean (Vigna angularis) to preplant-incorporated herbicides. Weed Technol., v. 19, n. 4, p. 897-901, 2005
VANGESSEL, M. J.; MONKS, D. W.; JOHNSON, Q. R. Herbicides for potential use in lima bean (Phaseolus lunatus) production. Weed Technol., v. 14, n. 2, p. 279-286, 2000

VICTORIA FILHO, R.; GODOY JÚNIOR, C. Herbicidas na cultura do feijão (Phaseolus vulgaris L.): controle, fitotoxicidade e persistência no solo. Planta Daninha, v. 1, n. 1, p. $25-37,1978$

VIGER, P. R.; EBERLEIN, C. V.; FUERST, E. P. Influence of available soil water content, temperature and CGA-154281 on metolachlor injury to corn. Weed Sci., v. 39, n. 2 , p. 227-231, 1991.

WILSON, R. G. Response of dry bean and weeds to fomesafen and fomesafen tank mixtures. Weed Technol., v. 19, n. 1, p. $201-206,2005$ 